\title{
Determination of the Creep Parameters of Linear Viscoelastic Materials
}

\author{
Alibay Iskakbayev, ${ }^{1}$ Bagdat Teltayev, ${ }^{2}$ and Sergei Alexandrov ${ }^{3}$ \\ ${ }^{1}$ Department of Mechanics, Al-Farabi Kazakh National University, 71 Al-Farabi, Almaty 050040, Kazakhstan \\ ${ }^{2}$ Kazakhstan Highway Research Institute, 2 A Nurpeisov, Almaty 050061, Kazakhstan \\ ${ }^{3}$ Institute for Problems in Mechanics, Russian Academy of Sciences, 101 Prospect Vernadskogo, Moscow 117526, Russia \\ Correspondence should be addressed to Bagdat Teltayev; bagdatbt@yahoo.com
}

Received 9 March 2016; Accepted 7 June 2016

Academic Editor: M. Shariyat

Copyright (C) 2016 Alibay Iskakbayev et al. This is an open access article distributed under the Creative Commons Attribution License, which permits unrestricted use, distribution, and reproduction in any medium, provided the original work is properly cited.

\begin{abstract}
Creep process of linear viscoelastic materials is described by the integral equation of Boltzmann-Volterra in which creep kernel is approximated by Rabotnov's fractional exponential function. The creep equation contains four unknown parameters: $\alpha$, singularity parameter; $\beta$, fading parameter; $\lambda$, rheological parameter; and $\varepsilon_{0}$, conditionally instantaneous strain. Two-stage determination method of creep parameters is offered. At the first stage, taking into account weak singularity properties of Abel's function at the initial moment of loading, parameters $\varepsilon_{0}$ and $\alpha$ are determined. At the second stage, using already known parameters $\varepsilon_{0}$ and $\alpha$, parameters $\beta$ and $\lambda$ are determined. Analytical expressions for calculating these parameters are obtained. An accuracy evaluation of the offered method with using experimentally determined creep strains of material Nylon 6 and asphalt concrete showed its high accuracy.
\end{abstract}

\section{Introduction}

Many materials used in technique and production (rocks, soils, metals, concretes, polymers, composites, etc.) under loading show viscoelastic properties. Currently a number of methods for quantitative evaluation of viscoelastic properties of materials are known [1-4]. One of the most frequently used methods is creep testing under uniaxial stress [5]. In the mathematical description of creep process of linear viscoelastic material the integral equation of Boltzmann-Volterra with corresponding creep kernel is widely used. A large number of analytical expressions for the creep kernel are known $[1,4,5]$.

In testing samples of viscoelastic materials on creep, usually it is difficult to measure instantaneous elastic strain $\varepsilon_{0}=$ $\varepsilon(t=0)$. This is due to the fact that in the initial time after application of load to sample the rate of creep is extremely high. Its accurate measurement is practically impossible due to the dynamic effects caused by inertia of loading devices and measuring equipment.

Creep process of many materials particularly at small loads and low temperatures proceeds for a very long time. At high times of loading the rate of creep starts to fade and strain slowly approaching asymptotic value.

Thus, we can assume that creep kernel of integral equation must have the following two properties: firstly, having a weak singularity at the initial time point and, secondly, having a property of exponential function at high times of loading.

The most universal description of viscoelastic properties of materials satisfying the above requirements is a kernel in the form of fractional exponential function of Rabotnov [47]. Fractional exponential function is well studied and for simplification of calculations by using it, a special table has been developed [8]. Another advantage of the fractional exponential function is that for known creep kernel parameters constructed with using the fractional exponential function a kernel of relaxation becomes definite $[9,10]$.

Some methods for determining the parameters of Rabotnov's creep kernel are known: using Mittag-Leffler's function $[11,12]$, using Laplace-Carson's transform [9, 12-14], and a method of direct approximation [9, 12-15].

In this paper we offer a new double stage method for determining the creep kernel parameters of linear viscoelastic 


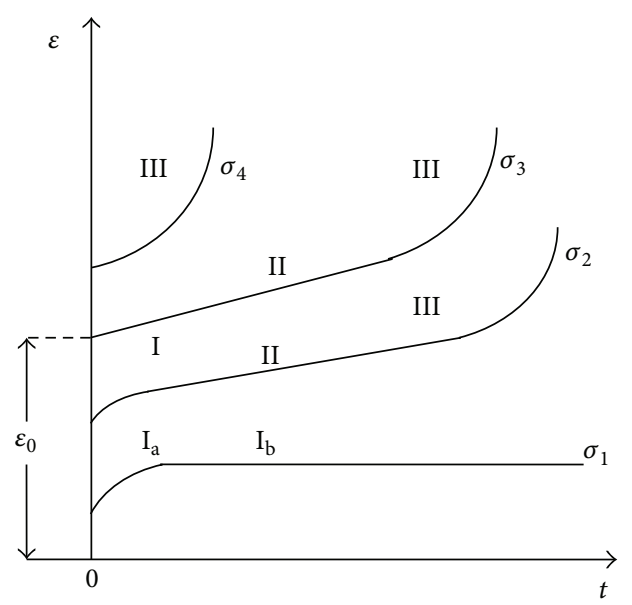

FIGURE 1: Various types of creep curves.

materials constructed with using Rabotnov's fractional exponential function. At the first stage, taking into account weak singularity properties of Abel's function at the initial moment of loading, parameters $\varepsilon_{0}$ and $\alpha$ are determined. At the second stage, using already known parameters $\varepsilon_{0}$ and $\alpha$, parameters $\beta$ and $\lambda$ are determined.

Fractional derivative constitutive models for finite deformation of viscoelastic materials developed with using a kernel close to Abel's kernel are given in [16]. A new model for the linear theory of generalized thermoviscoelasticity using a relaxation kernel close to Abel's kernel is demonstrated in [17].

\section{Creep Equation and Kernel}

As is known generally creep curve of the viscoelastic material has three characteristic sites: I, site with a decreasing rate of creep (unstabilized creep), II, site with a constant (minimum) rate of creep (stabilized creep), and III, site of accelerating creep, preceding failure (Figure 1).

In the present work sites I and II of creep curves of linear viscoelastic materials under uniaxial tension are described by the integral equation of Boltzmann-Volterra:

$$
\varepsilon(t)=\frac{1}{E}\left[\sigma(t)+\int_{0}^{t} K(t-\tau) \sigma(\tau) d \tau\right],
$$

where $\varepsilon(t)$ is strain at time point $t, \sigma(t), \sigma(\tau)$ are stresses at time points $t$ and $\tau, K(t-\tau)$ is creep kernel, $E$ is instant modulus of elasticity, $t$ is observation time, and $\tau$ is time, preceding observation time.

Taking into account that for creep process $\sigma_{0}=$ const, from (1) we will find

$$
\varepsilon(t)=\varepsilon_{0}\left[1+\int_{0}^{t} K(t-\tau) d \tau\right]
$$

where $\varepsilon_{0}$ is conditionally instantaneous strain.

In (2) it was accounted that on Hooke's law $\sigma_{0}=E \cdot \varepsilon_{0}$.
As mentioned above, creep kernel recorded using Rabotnov's fractional exponential function in the following form [6]:

$$
\begin{aligned}
K(t-\tau) & =\lambda \ni_{-\alpha}(-\beta, t-\tau) \\
& =\lambda(t-\tau)^{-\alpha} \sum_{n=0}^{\infty} \frac{(-\beta)^{n}(t-\tau)^{(1-\alpha) n}}{\Gamma[(1-\alpha)(1+n)]},
\end{aligned}
$$

where $\ni_{-\alpha}(-\beta, t-\tau)$ is Rabotnov's fractional exponential function, $\alpha, \beta$, and $\lambda$ are creep kernel parameters $(\lambda>0$, $0<\alpha<1$, and $\beta>0$ ) and $\Gamma$ is gamma-function.

Substituting expression (3) for creep kernel in the creep equation (2) we obtain the following equation:

$$
\varepsilon(t)=\varepsilon_{0}\left[1+\lambda \sum_{n=0}^{\infty} \frac{(-\beta)^{n} t^{(1-\alpha)(1+n)}}{\Gamma[(1-\alpha)(1+n)+1]}\right] .
$$

Equation (4) represents creep equation recorded using Rabotnov's fractional exponential function. It contains four unknown parameters: $\alpha$ is the singularity parameter; $\beta$ is the attenuation parameter; $\lambda$ is the rheological parameter; and $\varepsilon_{0}$ is conditionally instantaneous strain.

\section{Method of Determining the Creep Parameters}

3.1. Parameters $\varepsilon_{0}$ and $\alpha$. Taking $n=0$ from series (4) we find the first term:

$$
\varepsilon(t)=\varepsilon_{0}\left[1+\frac{\delta}{1-\alpha} t^{1-\alpha}\right]
$$

As one can see, obtained expression contains well-known Abel's function with parameter $\delta>0$. Abel's function at $t=0$ has a singularity at order $\alpha$. Based on this property of Abel's function, unknown parameters $\varepsilon_{0}$ and $\delta$ are determined by use of (5).

Knowing that parameter $\alpha$ takes value from interval $(0,1)$ we will consider as unknown only parameters $\varepsilon_{0}$ and $\delta$.

According to the least squares method the best values of parameters $\varepsilon_{0}$ and $\delta$ are those for which the following condition is met:

$$
S\left(\varepsilon_{0}, \delta\right)=\sum_{i=1}^{m}\left[\varepsilon_{0}\left(1+\frac{\delta}{1-\alpha} t_{i}^{1-\alpha}\right)-\varepsilon_{\mathrm{ei}}\right]^{2} \longrightarrow \min ,
$$

where $S\left(\varepsilon_{0}, \delta\right)$ is sum of squares of deviations, $\varepsilon_{\mathrm{ei}}$ is values of creep strain determined experimentally, and $m$ is number of creep strains.

From two equations based on expressions $\partial S\left(\varepsilon_{0}, \delta\right) / \partial \varepsilon_{0}=$ 0 and $\partial S\left(\varepsilon_{0}, \delta\right) / \partial \delta=0$, we find expressions for determining the parameters $\varepsilon_{0}$ and $\delta$ :

$$
\begin{aligned}
\varepsilon_{0} & =\frac{\sum_{i=1}^{m} \varepsilon_{i} \sum_{i=1}^{m} t_{i}^{2(1-\alpha)}-\sum_{i=1}^{m} t_{i}^{(1-\alpha)} \sum_{i=1}^{m} \varepsilon_{1} t_{i}^{(1-\alpha)}}{m \sum_{i=1}^{m} t_{i}^{2(1-\alpha)}-\left[\sum_{i=1}^{m} t_{i}^{(1-\alpha)}\right]^{2}}, \\
\delta & =\frac{\sum_{i=1}^{m}\left(\varepsilon_{i} / \varepsilon_{0}-1\right) t_{i}^{(1-\alpha)}}{(1 /(1-\alpha)) \sum_{i=1}^{m} t_{i}^{2(1-\alpha)}} .
\end{aligned}
$$


Setting values of parameter $\alpha$ from interval $(0,1)$ with a certain step from expression (7) we will find values of parameter $\varepsilon_{0}=\varepsilon_{0}(\alpha)$. Substituting the found values of parameter $\varepsilon_{0}$ and the corresponding values of singularity parameter $\alpha$ in expression (8) values of parameter $\delta=\delta\left(\varepsilon_{0}, \alpha\right)$ are determined.

Further, sequentially substituting values of singularity parameter $\alpha$ and calculated corresponding values of parameters $\varepsilon_{0}$ and $\delta$ in expression (5), values of creep strain $\varepsilon\left(t_{i}\right)=$ $\varepsilon\left(t_{i}, \alpha, \varepsilon_{0}, \delta\right)$ are calculated.

If we designate an average deviation of calculated values of creep strains from experimental values through $\Delta \varepsilon_{m}(\lambda$, $\varepsilon_{0}, \delta$ ), as a selecting criterion of the best values of parameters $\alpha, \varepsilon_{0}$, and $\delta$ the following condition can be accepted:

$$
\Delta \varepsilon_{\mathrm{mi}}\left(\alpha_{i}, \varepsilon_{0 i}, \delta_{i}\right) \longrightarrow \min .
$$

3.2. Parameters $\beta$ and $\lambda$. Rewrite equation of creep (4) in the following form:

$$
\begin{aligned}
\varepsilon(t) & =\varepsilon_{0}\left(1+\lambda t^{1-\alpha} F_{2 i}\right), \\
\text { where } F_{2 i} & =\sum_{n=0}^{\infty} \frac{(-\beta)^{n} t^{(1-\alpha) n}}{\Gamma[(1-\alpha)(1+n)+1]} .
\end{aligned}
$$

According to the least squares method analogically to condition (6) we write an extremum condition using the equation of creep (10):

$$
S(\beta, \lambda)=\sum_{i=1}^{m}\left[\varepsilon_{0}\left(1+\lambda t_{i}^{1-\alpha} F_{2 i}\right)-\varepsilon_{\mathrm{ei}}\right]^{2} \longrightarrow \min .
$$

From two equations based on the expressions $\partial S(\beta, \lambda) /$ $\partial \beta=0$ and $\partial S(\beta, \lambda) / \partial \lambda=0$, expressions for determining the parameters $\beta$ and $\lambda$ are found:

$$
\begin{aligned}
& \sum_{i=1}^{m}\left[\left(1-\frac{\varepsilon_{i}}{\varepsilon_{0}}\right) \sum_{i=1}^{m}\left(t_{i}^{1-\alpha} F_{2 i}\right)^{2}\right. \\
& \left.\quad+t_{i}^{1-\alpha} F_{2 i} \sum_{i=1}^{m}\left(\frac{\varepsilon_{i}}{\varepsilon_{0}}-1\right) t_{i}^{1-\alpha} F_{2 i}\right]\left(t_{i}^{1-\alpha}\right)^{2} F_{3 i}=0, \\
& \lambda=\frac{\sum_{i=1}^{m}\left(\varepsilon_{i} / \varepsilon_{0}-1\right) t_{i}^{1-\alpha} F_{2 i}}{\sum_{i=1}^{m}\left(t_{i}^{1-\alpha} F_{2 i}\right)^{2}} \\
& \text { where } F_{3 i}=\sum_{n=1}^{\infty}(-1)^{n} \frac{n(\beta)^{n-1} t_{i}^{(1-\alpha)(n-1)}}{\Gamma[(1-\alpha)(n+1)+1]},
\end{aligned}
$$

but series $F_{21}$ is determined by expression (11).

Value of parameter of fading $\beta$ is determined from (13) by substituting trial values. If (13) has a unique solution, it will be desired value of the parameter $\beta>0$. Obviously, for determining the values of parameters $\beta$ and $\lambda$ from expressions (13) and (14) earlier calculated values of parameters $\varepsilon_{0}$ and $\alpha$ are used.
TABLE 1: Strains in site I of material Nylon 6 creep curve.

\begin{tabular}{lccc}
\hline Time $t, h$ & \multicolumn{2}{c}{ Creep strain (\%) calculated } & Deviation, \% \\
& $(16)$ & $(17)$ & \\
\hline 0,01 & 0,2919 & 0,2957 & $+1,30$ \\
0,03 & 0,3184 & 0,3186 & $+0,06$ \\
0,05 & 0,3315 & 0,3306 & $-0,27$ \\
0,07 & 0,3404 & 0,3389 & $-0,44$ \\
0,09 & 0,3472 & 0,3455 & $-0,49$ \\
0,10 & 0,3501 & 0,3483 & $-0,51$ \\
0,30 & 0,3819 & 0,3806 & $-0,34$ \\
0,50 & 0,3976 & 0,3975 & $-0,01$ \\
0,70 & 0,4083 & 0,4094 & $+0,27$ \\
0,90 & 0,4165 & 0,4187 & $+0,53$ \\
\hline
\end{tabular}

\section{Experimental Approbation}

4.1. Material Nylon 6. In work [9] the creep curve of Nylon 6 at a stress of $\sigma_{0}=5 \mathrm{MPa}$ was approximated by expression

$$
\varepsilon_{e}(t)=0,42 t^{0,079},
$$

where $\varepsilon[\%]$ is strain and $t[\mathrm{~h}]$ is time.

Firstly, for determination of parameters $\varepsilon_{0}$ and $\alpha$ we calculate some strain values in time interval $t=0,01 \cdots 0,9 \mathrm{~h}$. Time values and corresponding creep strain values are given in the first and the second columns of Table 1 .

Substituting values of time $t$, strain $\varepsilon$, and various values of singularity parameter $\alpha$ from interval $0,05 \cdots 0,95$ with step of 0,05 in expressions (7) and (8) and taking into account condition (9) it has been found that $\alpha=0,85 ; \varepsilon_{0}=0,1682$; $\delta=0,2269$.

With consideration of found values of parameters $\alpha, \varepsilon_{0}$, and $\delta$, (5) constructed using Abel's function for describing strain in site I of creep curve takes the form

$$
\varepsilon(t)=0,1682\left(1+1,5127 t^{0,15}\right) .
$$

Calculated by (17) values of creep strain and corresponding approximation errors are presented in the third and the fourth columns of Table 1 .

As can be seen from Table 1, (17) with parameters $\varepsilon_{0}$ and $\alpha$ found by the offered method has high accuracy of approximation.

Since the parameter $\beta$ characterizes rate of material creep strain at long times, for definition it and parameter $\lambda$ consider creep process of material Nylon 6 in the time interval $t=$ $0,01 \cdots 100 \mathrm{~h}$. Time values and corresponding values of creep strain calculated according to expression (16) are given in the first and the second columns of Table 2.

Using values of time $t$, strain $\varepsilon$, parameters $\alpha=0,85, \varepsilon_{0}=$ 0,1682 , and trial values of parameter $\beta>0$, from expression (13) it has been found that $\beta=0,18$. Further from expression (14) we will define $\lambda=1,6682$. 
TABLE 2: Strains in sites $I_{a}$ and $I_{b}$ of material Nylon 6 creep curve.

\begin{tabular}{|c|c|c|c|}
\hline \multirow[t]{2}{*}{ Time $t, h$} & \multicolumn{2}{|c|}{$\begin{array}{c}\text { Creep strain (\%) calculated } \\
\text { by expression }\end{array}$} & \multirow[t]{2}{*}{ Deviation, \% } \\
\hline & (16) & $(18)$ & \\
\hline 0,01 & 0,2919 & 0,3060 & $+4,83$ \\
\hline 0,03 & 0,3184 & 0,3282 & $+3,08$ \\
\hline 0,05 & 0,3315 & 0,3396 & $+2,44$ \\
\hline 0,07 & 0,3404 & 0,3474 & $+2,06$ \\
\hline 0,09 & 0,3472 & 0,3535 & $+1,82$ \\
\hline 0,1 & 0,3501 & 0,3561 & $+1,72$ \\
\hline 0,3 & 0,3819 & 0,3852 & $+0,86$ \\
\hline 0,5 & 0,3976 & 0,3999 & $+0,58$ \\
\hline 0,7 & 0,4083 & 0,4102 & $+0,46$ \\
\hline 0,9 & 0,4165 & 0,4180 & $+0,36$ \\
\hline 1 & 0,4200 & 0,4214 & $+0,33$ \\
\hline 3 & 0,4581 & 0,4584 & $+0,07$ \\
\hline 5 & 0,4769 & 0,4770 & $+0,02$ \\
\hline 7 & 0,4898 & 0,4898 & 0,00 \\
\hline 9 & 0,4996 & 0,4996 & 0,00 \\
\hline 10 & 0,5038 & 0,5038 & 0,00 \\
\hline 20 & 0,5321 & 0,5321 & 0,00 \\
\hline 30 & 0,5495 & 0,5495 & 0,00 \\
\hline 40 & 0,5621 & 0,5621 & 0,00 \\
\hline 50 & 0,5721 & 0,5721 & 0,00 \\
\hline 60 & 0,5804 & 0,5804 & 0,00 \\
\hline 70 & 0,5875 & 0,5875 & 0,00 \\
\hline 80 & 0,5937 & 0,5937 & 0,00 \\
\hline 90 & 0,5993 & 0,5992 & 0,00 \\
\hline 100 & 0,6043 & 0,6042 & 0,00 \\
\hline
\end{tabular}

After substitution values of the found parameters $\varepsilon_{0}, \alpha, \beta$, and $\lambda(10)$ and auxiliary function $F_{2 i}(11)$ will have the next form:

$$
\begin{aligned}
\mathcal{E}(t) & =0,1682\left(1+1,6682 t^{0,15} F_{2 i}\right), \\
F_{2 i} & =\sum_{n=0}^{\infty} \frac{(-0,18)^{n} t^{0,15 n}}{\Gamma[0,15(1+n)+1]} .
\end{aligned}
$$

Calculated by (18) values of creep strain and corresponding errors of approximation are given in the last two columns of Table 2.

As can be seen from Table 2 creep (18) with parameters $\varepsilon_{0}$, $\alpha, \beta$, and $\lambda$ defined by the offered method has high accuracy of approximation.

4.2. Asphalt Concrete. The strains calculated by approximation formula (16) were taken for determination of the above creep parameters of material Nylon 6 instead of strains obtained experimentally. The reason for this was the fact that in work [9] the creep strains were given only graphically in the form of a creep curve and numerical values of the strain are not shown. It appeared almost impossible to find the strain

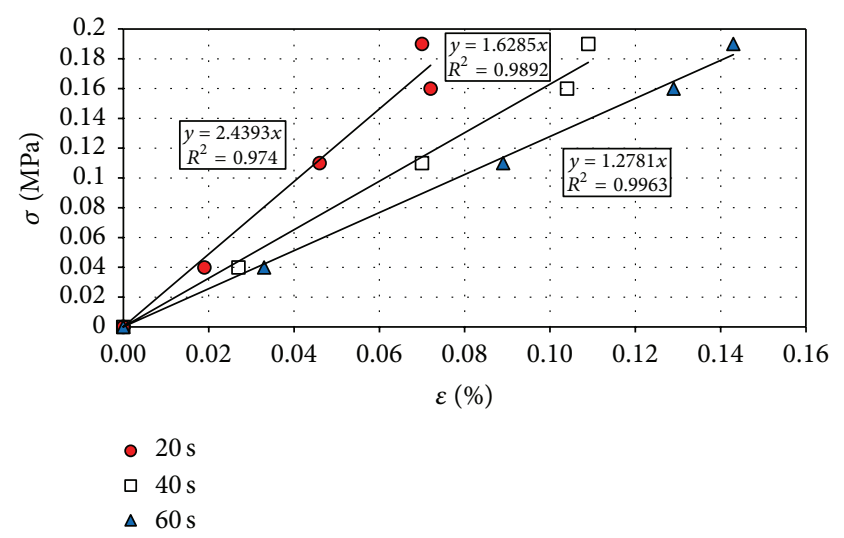

FIGURE 2: Isochronous lines of deformation of asphalt concrete.

values from the graphical creep curve with sufficient accuracy especially at short times of loading.

Possibly, in order to reduce the length of material in published works containing an approximation of creep curves of various materials, as a rule, information on creep strain is given graphically, for example, in works [9, 10, 12-14, 18-20].

Further, we will show determination of creep parameters $\varepsilon_{0}$ and $\alpha$ by the above offered method (expressions (7) and (8)) using experimentally determined values of strain at site I of creep of hot fine-grained asphalt concrete. This type of asphalt is used for application on top layer of road surface in many countries, including Kazakhstan. Asphalt concrete mix that meets the requirements of the Kazakhstan standard ST RK 1225 [21] was prepared with use of viscous road bitumen of grade BND 100/130 from Pavlodar petrochemical plant, which meets the standard requirements of ST RK 1373 [22]. Samples of asphalt concrete with rectangular cross-section dimensions of $5 \times 5 \times 15 \mathrm{~cm}$ were prepared by means of Cooper compactor (UK, model CRT-RC2S) in accordance with European Union standard EN 12697-33 [23]. Detailed information on preparation of asphalt mixtures and samples, as well as standard properties of bitumen and asphalt concrete, is given in [24]. Samples of asphalt concrete were tested for creep according to the scheme of uniaxial tension at a temperature of $20 \pm 2^{\circ} \mathrm{C}$ and different loads.

Isochronous lines of deformation for the asphalt concrete constructed using creep curves within site I at durations of loadings 20,40, and $60 \mathrm{~s}$ are shown in Figure 2. As it can be seen dependence between stress and strain is linear; that is, asphalt concrete under these conditions is linearly deformable material and for description of its creep process the equation of Boltzmann-Volterra is applicable.

Figures 3 and 4 show site I of creep curves of asphalt concrete at stresses of 0.16 and $0.19 \mathrm{MPa}$, respectively. In these figures points designate the creep strain defined experimentally and lines designate its approximation by Abel's kernel (5). It is seen that compliance of approximating curves to the experimental data is quite good. Average deviations of calculated strains from experimental creep curves at 0,16 and $0,19 \mathrm{MPa}$ are $1.5 \%$ and $0.5 \%$, respectively. The values of parameters $\varepsilon_{0}$ and $\alpha$ found by the offered method are equal to 


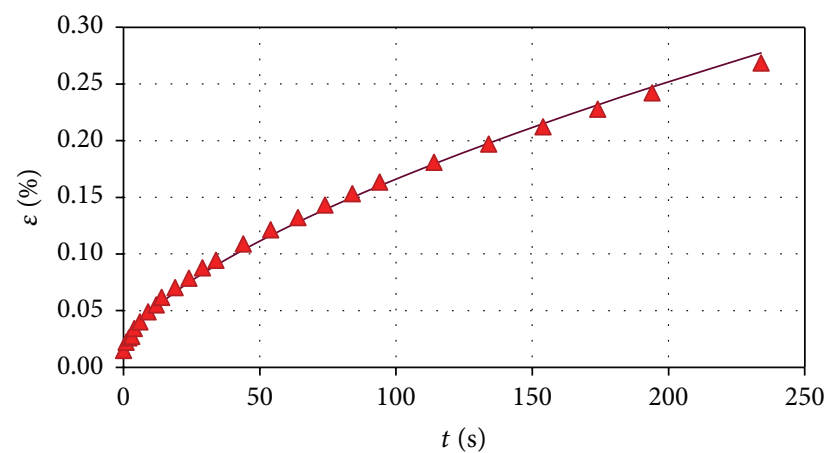

$\Delta$ Experiment

- Approximation

FIGURE 3: Creep curve of asphalt concrete at stress $\sigma_{0}=0,16 \mathrm{MPa}$.

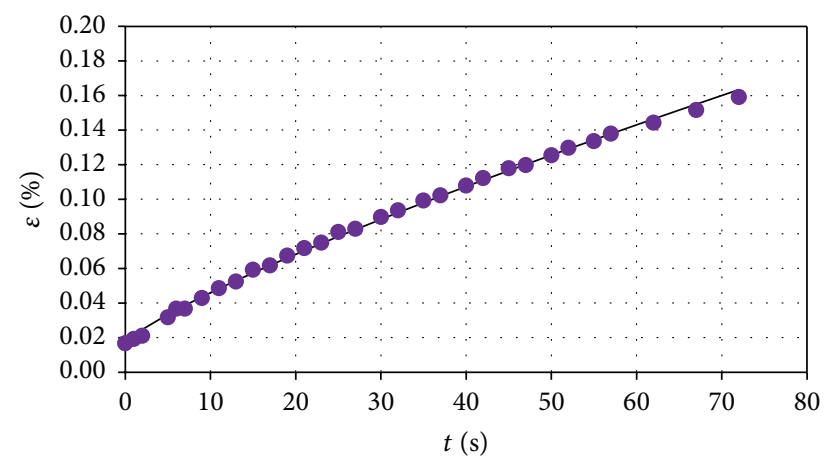

- Experiment

- Approximation

FIGURE 4: Creep curve of asphalt concrete at stress $\sigma_{0}=0,19 \mathrm{MPa}$.

$\varepsilon_{0}=0,015217 \%$ and $\alpha=0,35$ at $\sigma_{0}=0,16 \mathrm{MPa}$ and $\varepsilon_{0}=$ $0,016871 \%$ and $\alpha=0,18$ at $\sigma_{0}=0,19 \mathrm{MPa}$.

Thus, the integral equation of Boltzmann-Volterra with creep kernel constructed using Rabotnov's fractional exponential function with parameters defined by the offered method describes the creep process of linear viscoelastic materials with high accuracy.

\section{Conclusions}

(1) Describing the creep process of linear viscoelastic materials by the integral equation of BoltzmannVolterra with creep kernel which is constructed using Rabotnov's fractional exponential function is suggested.

(2) A new method of determining the creep kernel parameters of linear viscoelastic materials constructed using Rabotnov's fractional exponential function is offered. It is implemented in two stages. At the first stage based on the property of singularity of Abel's function at the initial moment of time parameters $\varepsilon_{0}$ and $\alpha$ are defined. At the second stage using already known parameters $\varepsilon_{0}$ and $\alpha$ parameters $\beta$ and $\lambda$ are defined. Analytical expressions for calculating these parameters are obtained.

(3) Applicability of the offered method is evaluated using data on creep strain of material Nylon 6 and hot finegrained asphalt concrete obtained experimentally. The results showed high accuracy of the offered method.

\section{Competing Interests}

The authors declare that they have no competing interests.

\section{References}

[1] R. M. Cristensen, Theory of Viscoelasticity: An Introduction, Academic Press, New York, NY, USA, 1971.

[2] N. W. Tschoegl, The Phenomenological Theory of Linear Viscoelastic Behavior: An Introduction, Springer, Berlin, Germany, 1989.

[3] J. D. Ferry, Viscoelastic Properties of Polymers, John Wiley \& Sons, New York, NY, USA, 3rd edition, 1980.

[4] Yu. N. Rabotnov, Mechanics of Deformed Solid Body, Nauka, Moscow, Russia, 1988.

[5] Yu. N. Rabotnov, Creep of Structure Elements, Nauka, Moscow, Russia, 1966.

[6] Y. N. Rabotnov, Elements of Hereditary Mechanics of Solids, Nauka, Moscow, Russia, 1977.

[7] Yu. N. Rabotnov, "Balance of the elastic medium with a aftereffect," Applied Mathematics and Mechanics, vol. 12, pp. 53$62,1948$.

[8] Y. N. Rabotnov, L. Kh. Papernik, and E. N. Zvonov, Tables of a Fractional Exponential Function of Negative Parameter and Its Integral, Nauka, Moscow, Russia, 1969.

[9] Yu. V. Suvorova, “About Yu.N. Rabotnov's nonlinear hereditary equation and its applications," News of the Russian Academy of Sciences, Mechanics of solids, no. 1, pp. 174-181, 2004.

[10] Y. V. Suvorova, "Master equations for viscoelastic materials taking into account the influence of temperature and humidity," Strength of Materials, vol. 32, no. 5, pp. 490-496, 2000.

[11] M. I. Rozovskii, "Some features of elasticity hereditary media," News of the USSR Academy of Sciences, no. 2, pp. 30-36, 1961.

[12] V. P. Golub, P. V. Fernati, and Y. G. Lyashenko, "Determining the parameters of the fractional exponential heredity kernels of linear viscoelastic materials," International Applied Mechanics, vol. 44, no. 9, pp. 963-974, 2008.

[13] E. N. Zvonov, N. I. Malinin, L. Kh. Papernik, and B. M. Tseitlin, "Determination of the creep characteristics of linear elastic hereditary materials by using a digital computer," Mechanics of Solid, no. 5, pp. 76-82, 1968.

[14] Yu. V. Suvorova and A. V. Mosin, "Determination of parameters of the Rabotnov's fractional exponential function with use of integral transform and modern software," Problems of Mechanical Engineering and Automation, vol. 4, pp. 54-56, 2002.

[15] D. A. Gavrilov and V. A. Markov, "Numerical method of determining the rheologic parameters of composites from test results," Mechanics of Composite Materials, vol. 22, no. 4, pp. 417-421, 1987.

[16] M. Fukunaga and N. Shimizu, "Fractional derivative constitutive models for finite deformation of viscoelastic materials," 
Journal of Computational and Nonlinear Dynamics, vol. 10, no. 6, Article ID 061002, 8 pages, 2015.

[17] M. A. Ezzat, A. S. El-Karamany, and A. A. El-Bary, "Thermoviscoelastic materials with fractional relaxation operators," Applied Mathematical Modelling, vol. 39, no. 23-24, pp. 74997512, 2015.

[18] R. D. Maksimov, L. Jirgens, J. Jansons, and E. Plume, "Mechanical properties of polyester polymer-concrete," Mechanics of Composite Materials, vol. 35, no. 2, pp. 99-110, 1999.

[19] R. D. Maksimov and E. Plume, "Long-term creep of hybrid aramid/glass-fiber-reinforced plastics," Mechanics of Composite Materials, vol. 37, no. 4, pp. 271-280, 2001.

[20] E. A. Sokolov and R. D. Maksimov, "Possibilities of predicting the creep of a polymeric fiber-reinforced plastic from the properties of the components," Polymer Mechanics, vol. 14, no. 6, pp. 807-813, 1978.

[21] ST RK 1225-2003, "Asphalt concrete mixes for road, airfield and asphalt concrete," Technical specifications, Astana, Kazakhstan, 2003.

[22] "Bitumen and bituminous binders. Oil road viscous bitumens," ST RK 1373-2005, Technical Specifications, Astana, Kazakhstan, 2005.

[23] European Committee for Standardization, "Bituminous mixtures. Test methods for hot mix asphalt - part 33: specimen prepared by roller compactor," Tech. Rep. EN 12697-33, 2003.

[24] B. Teltayev, "A new failure criterion for asphalt mixtures under fatigue loading," International Journal of Pavement Research and Technology, vol. 8, no. 4, pp. 276-282, 2015. 


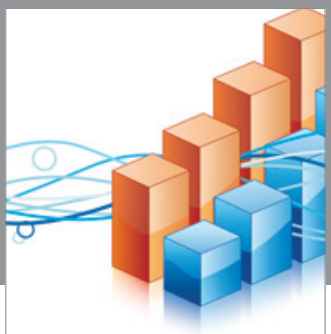

Advances in

Operations Research

vatem alat4

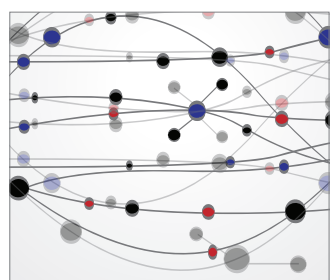

\section{The Scientific} World Journal
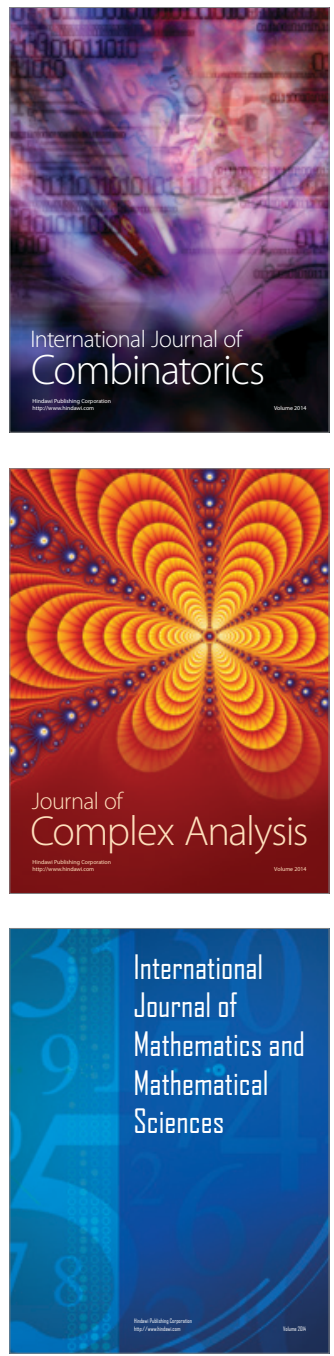
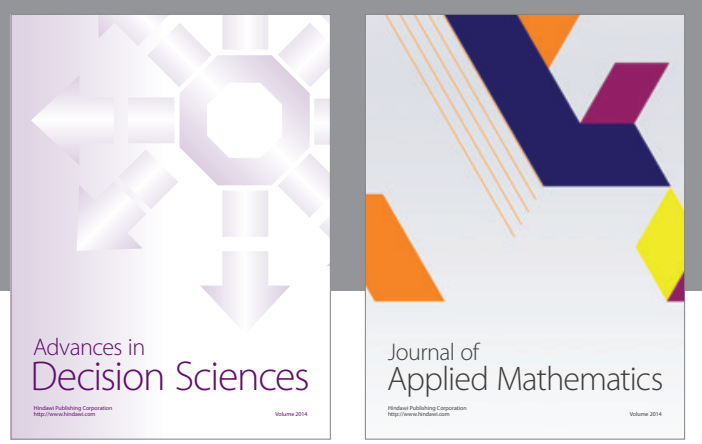

Algebra

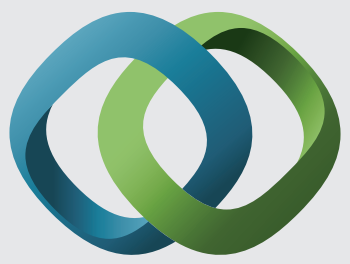

\section{Hindawi}

Submit your manuscripts at

http://www.hindawi.com
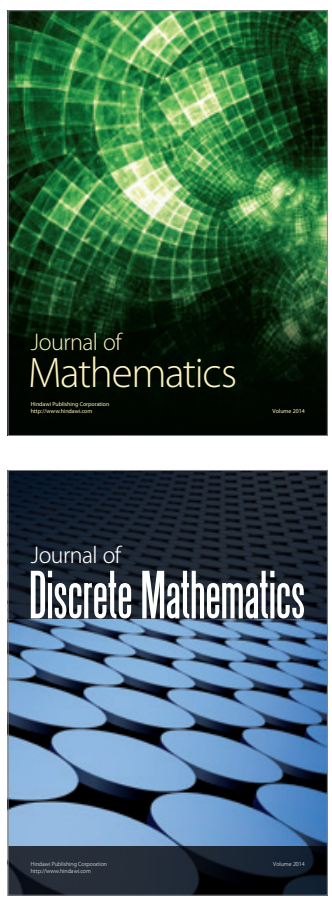

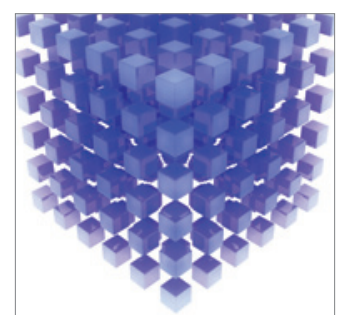

Mathematical Problems in Engineering
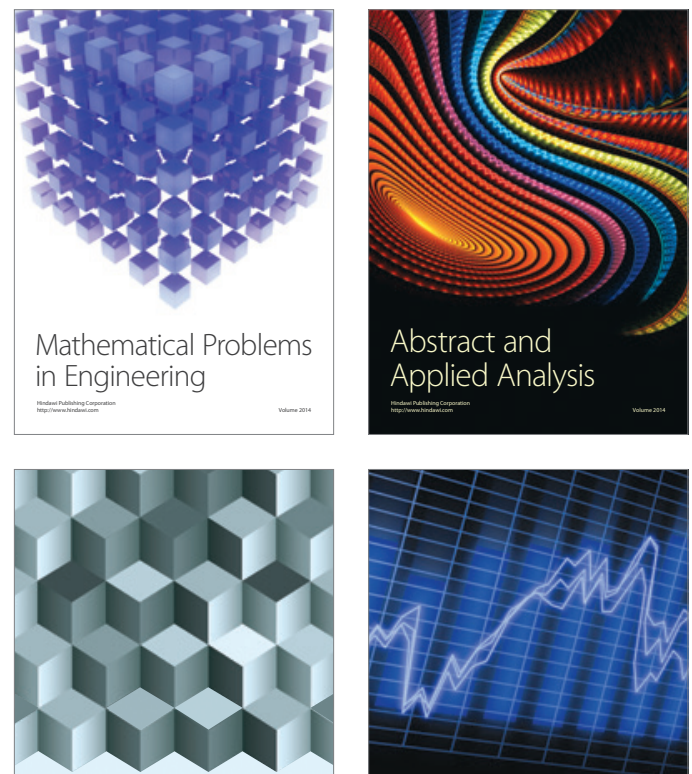

Journal of

Function Spaces

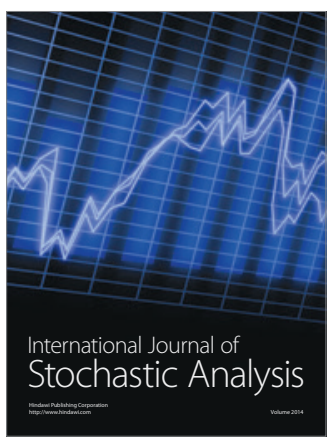

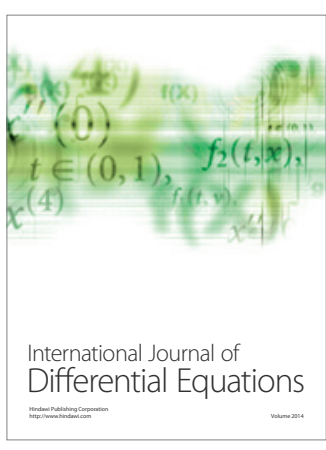
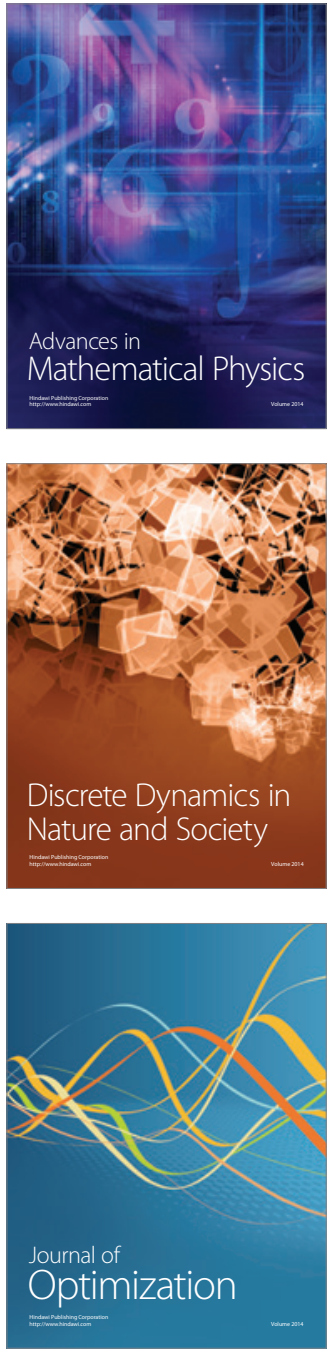